\title{
Efecto de diferentes niveles de proteína y lípidos totales en la dieta sobre el crecimiento y supervivencia de juveniles de pargo lunarejo Lutjanus guttatus
}

Effect of dietary and lipid level on growth performance and survival of juvenile spotted rose snapper Lutjanus guttatus

\author{
M$^{\text {a. Isabel Abdo de la Parra }}{ }^{1}$, L. Estela Rodríguez-Ibarra ${ }^{1}$, Crisantema \\ Hernández ${ }^{1}$, Karina Hernández ${ }^{1}$, Blanca González-Rodríguez ${ }^{1}$, \\ Irma Martínez-Rodríguez ${ }^{1}$ y Armando García-Ortega ${ }^{1}$
}

\begin{abstract}
${ }^{1}$ Centro de Investigación en Alimentación y Desarrollo, A. C., Unidad Mazatlán, Av. Sábalo Cerritos s/n. Mazatlán, Sinaloa 89010, México. abdo@ciad.mx
\end{abstract}

\begin{abstract}
The spotted rose snapper (Lutjanus guttatus) is one of the most commercially important fish species in México. As it is considered a suitable candidate for culture, then it is necessary to identify its nutritional requirements in order to succeed. The aim of this study was to evaluate the effects of different protein and lipid levels on growth and survival of juvenile spotted rose snappers. Nine semi-purified diets were formulated with three protein levels (40, 45 and 50\%) and three lipid levels (9, 12 and 15\%). Hatchery-produced juvenile spotted rose snappers $(2.2 \pm 0.1$ g) were used throughout the eight-week feeding experiment. Body weight, specific growth rate, condition factor, feed consumption, feed conversion rate, and survival were determined in each treatment at the end of the experiment. The highest weight gained and better feed conversion ratio were obtained in fish fed diets with 45 and $50 \%$ protein and their three lipids levels. Survival, feed consumption and condition factor were not affected by the treatments. These results indicate that juvenile spotted rose snappers require a minimum of $45 \%$ protein and $9 \%$ lipid for maximum growth and survival.
\end{abstract}

Key words: Nutritional requirements, practical feeds, marine fish

\begin{abstract}
Resumen. - El pargo lunarejo Lutjanus guttatus es una especie con un alto valor comercial en el mercado mexicano y con potencial para su cultivo. Por esto, es necesario determinar sus requerimientos nutricionales para desarrollar dietas específicas y adecuadas para el desarrollo de esta especie, actualmente inexistentes en el mercado mexicano. En el presente estudio, se evaluó el efecto de diferentes niveles de proteína y lípidos totales en la dieta sobre el crecimiento y supervivencia de juveniles de pargo lunarejos producidos en laboratorio. Se formularon nueve dietas semipuras con tres niveles de proteína $(40,45$ y 50\%) y tres niveles de lípidos $(9,12$ y $15 \%)$, con las cuales se alimentaron durante 8 semanas a los juveniles de pargo luranejo (peso promedio $=2,2 \pm 0,1 \mathrm{~g}$ ) producidos en criadero. Una vez finalizado el experimento, se determinó el incremento en peso, tasa específica de crecimiento, factor de condición, consumo de alimento, tasa de conversión alimenticia y porcentaje de supervivencia de los organismos. Los peces alimentados con las dietas de 45 y 50\% de proteína y sus tres niveles de lípidos obtuvieron mayor incremento en peso corporal y mejores tasa de conversión alimenticia que los organismos alimentados con las dietas de $40 \%$ de proteína y sus tres niveles de lípidos. La supervivencia, consumo de alimento y factor de condición no fueron afectados por los diferentes tratamientos. Estos resultados indican que los juveniles de pargo lunarejo requieren por lo menos $45 \%$ de proteína y $9 \%$ de lípidos totales en la dieta, para su mayor crecimiento y supervivencia.
\end{abstract}

Palabras clave: Requerimientos nutricionales, dietas prácticas, peces marinos

\section{INTRODUCCIÓN}

El pargo lunarejo, Lutjanus guttatus es un pez marino de gran importancia económica, tanto en México como en varios países de Latinoamérica, que se distribuye a lo largo de la costa del Pacífico del continente americano (RojasHerrera 2001). Se le considera una especie con alto potencial para su cultivo, debido a su demanda comercial, su capacidad de adaptación y control de su reproducción en cautiverio (Ibarra-Castro \& Duncan 2007). Además, se cuenta con la información biológica y los protocolos para producir juveniles en laboratorio (Boza-Abarca et al. 2008, Abdo de la Parra et al. 2010). Sin embargo, para desarrollar eficientemente las estrategias de cultivo, se necesitan elaborar dietas prácticas para esta especie, actualmente inexistentes en el mercado mexicano; para lo cual, es 
imprescindible determinar sus requerimientos nutricionales. Siendo el pargo un pez carnívoro, que se alimenta principalmente de peces y crustáceos (RojasHerrera 2001), sus requerimientos de proteína y lípidos deben ser altos.

La proteína es uno de los nutrientes más importantes para el crecimiento y es el macro componente más costoso en las dietas de peces carnívoros ya que se requiere en altas cantidades (NRC 1993). Los experimentos para determinar los requerimientos de proteína para especies acuáticas, casi siempre se realizan para establecer la cantidad mínima requerida de proteína para producir el máximo crecimiento, y no para ser utilizada como fuente de energía (Catacutan et al. 2001). La tendencia actual, en los alimentos para peces, es incrementar el contenido de lípidos para que sean éstos los que se utilicen como fuente energética y, por lo tanto; disminuir el nivel de proteína en la dieta, reducir costos y disminuir la cantidad de nitrógeno excretado por los peces (Schulz et al. 2008).

Los estudios sobre los requerimientos de proteína y lípidos para lutjánidos son escasos. Para el pargo lunarejo solo se han reportado para pre-adultos (100-145 g) capturados del medio natural, cuyo requerimiento se estableció en 40\% de proteína y 12\% lípidos (GarcíaOrtega 2009); sin embargo, no existen datos publicados para juveniles y reproductores. Para juveniles y sub-adultos del pargo rojo Lutjanus campechanus se reportó un requerimiento de proteína entre 32-36\% con 10\% de lípidos (Miller et al. 2005); para juveniles de pargo rojo de manglar L. argentimaculatus, se determinó un requerimiento de proteína de 44\% (Catacutan et al. 2001). Resultados similares se han reportado para el pargo del caribe, $L$. analis (Watanabe et al. 2001). Estos estudios han contribuido a desarrollar alimentos artificiales más adecuados y eficientes para el crecimiento de estas especies, que se cultivan en diferentes países de América y Asia.

De acuerdo a los antecedentes, es indispensable determinar los requerimientos nutricionales del pargo lugarejo, con el fin de desarrollar dietas para las distintas etapas productivas de esta especie y contribuir al desarrollo de la biotecnología de cultivo. En el presente trabajo se evaluó experimentalmente el efecto de diferentes niveles de proteína y lípidos en la dieta sobre el crecimiento, eficiencia alimenticia y supervivencia de juveniles de pargo lunarejo, Lutjanus guttatus.

\section{Material Y Métodos}

Se formularon 9 dietas semipuras, con tres niveles de proteína (40, 45 y 50\%) y cada una con 3 niveles de lípidos
(9, 12 y 15\%), utilizando como fuente proteica harina de pescado y caseína, y como fuente de lípidos, aceite de hígado de bacalao. El nivel de inclusión de las premezclas de minerales y vitaminas fue constante en las 9 dietas (Tabla 1). Para preparar las dietas, primero se mezclaron los macroingredientes en una batidora Kitchen Aid $^{\circledR}$ de $5 \mathrm{~L}$, después se añadieron los microingredientes, enseguida el aceite de hígado de bacalao y finalmente se agregó agua hasta obtener una mezcla homogénea. Para peletizar se utilizó un molino de carne Torrey ${ }^{\circledR}$ con un dado de $1 \mathrm{~mm}$. Los pellets se colocaron en bandejas para secarlos a $40^{\circ} \mathrm{C}$ durante $8 \mathrm{~h}$, se almacenaron en recipientes herméticos previamente etiquetados, y se mantuvieron en refrigeración a $4^{\circ} \mathrm{C}$. A los ingredientes y a las dietas experimentales, se les determinó su composición química en el laboratorio de Bromatología del CIAD Unidad Mazatlán, siguiendo los métodos de análisis proximal descrito por AOAC (2000). La proteína se evaluó por el método de combustión de Dumas (Ebling 1968), utilizando un equipo Leco FP528; el contenido de grasa se determinó mediante un equipo microSoxhlet; para determinar la humedad se utilizó una estufa Craft y las cenizas se obtuvieron por calcinación de la muestra en una mufla Felissa ${ }^{\circledR}$.

Los juveniles de pargo lunarejo se obtuvieron de la planta piloto de producción de peces marinos del CIAD Mazatlán, siguiendo los protocolos ya establecidos para el desove y cultivo larvario (Abdo de la Parra et al. 2010).

El bioensayo se realizó en las instalaciones del CIAD, Unidad Mazatlán, en 27 tanques circulares de fibra de vidrio color negro con una capacidad de $600 \mathrm{~L}$ cada uno, con un drenaje central de $50 \mathrm{~mm}$, cubierto con malla con una luz de 0,5 cm para evitar la salida de los peces y a la vez permitir la limpieza de los tanques. Cada tanque cuenta con aireación y flujo de agua continuo (6 $\mathrm{L} \mathrm{min}^{-1}$ aproximadamente), con distribuidores individuales para poder regularlos. El agua de mar es bombeada desde la playa Brujas en Mazatlán y pasa a través de filtros de arena y cartuchos de $16 \mu$ de retención relativa.

Se utilizó un diseño experimental factorial de 3 x 3 completamente al azar. En cada tanque experimental se colocaron 30 juveniles de pargo lunarejo, con un peso promedio de 2,2 $\pm 0,1 \mathrm{~g}$. Las dietas experimentales se distribuyeron manualmente cuatro veces al día, ofreciéndolas hasta saciedad aparente durante ocho semanas. Cada tratamiento se evaluó por triplicado. Diariamente, de cada tanque se registró la temperatura, salinidad y el oxígeno disuelto en el agua. Cada semana, el laboratorio de Química y Productividad Acuática del CIAD, Unidad Mazatlán, determinó el amonio presente 
Tabla 1. Ingredientes y composición proximal de las dietas experimentales para Lutjanus guttatus / Ingredients and proximal composition of experimental diets for Lutjanus guttatus

\begin{tabular}{|c|c|c|c|c|c|c|c|c|c|}
\hline $\begin{array}{l}\text { Ingrediente } \\
\left(\mathrm{g} \mathrm{Kg}^{-1}\right)\end{array}$ & 40P:9L & $40 \mathrm{P}: 12 \mathrm{~L}$ & $40 \mathrm{P}: 15 \mathrm{~L}$ & 45P:9L & $45 \mathrm{P}: 12 \mathrm{~L}$ & $45 \mathrm{P}: 15 \mathrm{~L}$ & 50P:9L & $50 \mathrm{P}: 12 \mathrm{~L}$ & $50 \mathrm{P}: 15 \mathrm{~L}$ \\
\hline Harina de pescado ${ }^{1}$ & 470,4 & 470,4 & 470,4 & 529,4 & 529,4 & 529,4 & 588,2 & 588,2 & 588,2 \\
\hline Caseína $^{2}$ & 88,8 & 88,8 & 88,8 & 100 & 100 & 100 & 111,1 & 111,1 & 111,1 \\
\hline Ac. hígado de bacalao ${ }^{3}$ & 49,9 & 79,9 & 109,9 & 44,9 & 74,9 & 104,9 & 39,9 & 69,9 & 99,9 \\
\hline Dextrina $^{3}$ & 369,8 & 337,8 & 307,8 & 302,9 & 272,9 & 242,9 & 237,9 & 207,9 & 177,9 \\
\hline Premezcla de minerales ${ }^{4}$ & 1 & 1 & 1 & 1 & 1 & 1 & 1 & 1 & 1 \\
\hline Premezcla de vitaminas ${ }^{4}$ & 1 & 1 & 1 & 1 & 1 & 1 & 1 & 1 & 1 \\
\hline Vitamina $C^{5}$ & 0,2 & 0,2 & 0,2 & 0,2 & 0,2 & 0,2 & 0,2 & 0,2 & 0,2 \\
\hline Cloruro de Colina ${ }^{6}$ & 0,5 & 0,5 & 0,5 & 0,5 & 0,5 & 0,5 & 0,5 & 0,5 & 0,5 \\
\hline Alginato $^{3}$ & 20 & 20 & 20 & 20 & 20 & 20 & 20 & 20 & 20 \\
\hline \multicolumn{10}{|c|}{ Contenido de nutrientes en base seca } \\
\hline Proteína (\%) & 41,4 & 40,4 & 41,0 & 45,7 & 45,5 & 45,6 & 53,5 & 52,6 & 50,1 \\
\hline Lípidos (\%) & 9,7 & 11,8 & 15,7 & 9,1 & 11,9 & 15,5 & 9,2 & 12,1 & 15,2 \\
\hline Cenizas (\%) & 8,4 & 8,6 & 8,2 & 9,5 & 9,5 & 10,0 & 10,2 & 11,2 & 11,7 \\
\hline $\begin{array}{l}\text { Relación proteína: } \\
\text { energía mg Kcal }{ }^{-1}\end{array}$ & 86,1 & 83,1 & 80,5 & 96,4 & 93,2 & 90,2 & 106,6 & 103,1 & 99,8 \\
\hline
\end{tabular}

$\mathrm{P}=\%$ de proteína en la dieta; $\mathrm{L}=\%$ de lípidos en la dieta; ${ }^{1}$ Proteinas Marinas Agropecuarias, S. A. de C.V; ${ }^{2}$ Sigma-Aldrich. St. Louis. MO. USA Libre de vitaminas, C3400-1KG; ${ }^{3}$ Droguería Cosmopolitan, S.A. de C.V.; ${ }^{4}$ Premezcla para peces marinos, elaborada por Empresa BASF. Mexicana; ${ }^{5}$ Vitamina $\mathrm{C}$ protegida donado por la empresa BASF. Mexicana; ${ }^{6}$ Donada por la empresa BASF. Mexicana

en el agua. Durante las ocho semanas del bioensayo la temperatura se mantuvo entre 29 y $29,8^{\circ} \mathrm{C}$, la salinidad varió de 32 a 34 ups y el oxígeno disuelto en el agua se mantuvo en un valor promedio de 4,5 $\pm 0,1 \mathrm{mg} \mathrm{L}^{-1}$ por tanque. El nitrógeno soluble total como indicador de calidad de agua en los tanques varió de 0,5 a 0,9 mg L-1.

Para determinar el crecimiento, los ejemplares fueron capturados con redes de cuchara y anestesiados con 2fenoxietanol (Sigma ${ }^{\circledR}$ ) a una concentración de $0,3 \mathrm{ml} \mathrm{L}^{-1}$, se pesaron individualmente en una balanza digital (precisión de $\pm 0,05$ g) y se determinó su longitud total (LT) con un ictiómetro.

Se calcularon los siguientes índices:

Incremento en peso $(\mathrm{g})$ :

IP = peso promedio final (g) - peso promedio inicial (g)

Tasa específica de crecimiento:

TEC $=100 \mathrm{x}$ (Ln peso final - Ln peso inicial $) /$ tiempo

Factor de condición:

$\mathrm{K}=\left[\left(\right.\right.$ Peso final $\left.\left./(\text { Largo total })^{3}\right)\right] \times 100$

Supervivencia (\%):

$\mathrm{S}=\mathrm{N}^{\circ}$ de peces final $/ \mathrm{N}^{\circ}$ de peces inicial x100

Tasa de conversión alimenticia:

TCA = Alimento consumido (g) / peso ganado (g)
Los datos en porcentaje fueron transformados en arcoseno para homogenizar las varianzas. Se verificó la normalidad y homogeneidad de los resultados obtenidos, al ser datos paramétricos se sometieron a un análisis de varianza factorial, ANDEVA de dos vías, mediante el programa Statgraphics Plus V.5,1. Cuando se encontraron diferencias significativas entre los tratamientos, se aplicó una prueba de rango múltiple de Tukey. La significancia estadística de todas las pruebas aplicadas fue considerada como $P<0,05$ (Zar 1996).

\section{Resultados}

Los parámetros de calidad del agua observados a lo largo del bioensayo se mantuvieron dentro de los rangos aceptables para el crecimiento del pargo lunarejo (Davis et al. 2000) y no se observaron signos patológicos de parásitos o de alguna enfermedad.

Al final de las ocho semanas de alimentación se determinó que el nivel de proteína en la dieta afectó significativamente el crecimiento independientemente del nivel de lípidos (Tabla 2); los juveniles alimentados con las dietas de 45 y $50 \%$ de proteína y sus tres niveles de lípidos presentaron mayor IP $\left(\mathrm{F}_{(2,18)}=16,78, P<0,0001\right)$ y mayores TEC $\left(\mathrm{F}_{(2,18)}=5,49, P=0,0137\right)$ que los pargos 
Tabla 2. Promedio \pm desviación estándar de crecimiento, supervivencia y eficiencia alimenticia de juveniles de Lutjanus guttatus alimentados con las dietas experimentales. Los promedios con distintos superíndices presentan diferencias significativas $(\mathbf{P}<\mathbf{0 , 0 5})$ / Productive performance, survival and feed efficiency of juvenile Lutjanus guttatus, fed on diets containing different protein and lipid levels. Means with different superscripts are significantly different $(P<0.05)$

\begin{tabular}{lccccccc}
\hline Dietas P:L & PI $(\mathrm{g})$ & IP $(\mathrm{g})$ & TEC $(\%)$ & $\mathrm{K}$ & $\mathrm{S}(\%)$ & $\mathrm{CA}(\mathrm{g})$ & TCA \\
\hline D-40:9 & $2,4 \pm 0,4$ & $15,6 \pm 1,4^{\mathrm{a}}$ & $3,47 \pm 0,4^{\mathrm{a}}$ & $1,6 \pm 0,02$ & $94,4 \pm 1,9$ & $30,1 \pm 1,6$ & $1,9 \pm 0,2^{\mathrm{b}}$ \\
D-40:12 & $2,1 \pm 0,1$ & $17,1 \pm 1,7^{\mathrm{a}}$ & $3,94 \pm 0,2^{\mathrm{a}}$ & $1,6 \pm 0,03$ & $91,1 \pm 7,7$ & $34,4 \pm 4,5$ & $2,0 \pm 0,4^{\mathrm{b}}$ \\
D-40:15 & $2,2 \pm 0,2$ & $15,8 \pm 0,2^{\mathrm{a}}$ & $3,74 \pm 0,1^{\mathrm{a}}$ & $1,6 \pm 0,02$ & $86,7 \pm 4,7$ & $39,2 \pm 6,4$ & $2,3 \pm 0,1^{\mathrm{c}}$ \\
D-45:9 & $2,2 \pm 0,1$ & $22,7 \pm 3,9^{\mathrm{b}}$ & $4,29 \pm 0,03^{\mathrm{b}}$ & $1,6 \pm 0,02$ & $97,8 \pm 1,9$ & $32,4 \pm 3,8$ & $1,4 \pm 0,1^{\mathrm{a}}$ \\
D-45:12 & $2,3 \pm 0,1$ & $22,8 \pm 1,3^{\mathrm{b}}$ & $4,19 \pm 0,1^{\mathrm{b}}$ & $1,6 \pm 0,03$ & $95,6 \pm 1,9$ & $34,7 \pm 0,9$ & $1,5 \pm 0,1^{\mathrm{a}}$ \\
D-45:15 & $2,2 \pm 0,3$ & $18,1 \pm 1,0^{\mathrm{ab}}$ & $4,00 \pm 0,1^{\mathrm{b}}$ & $1,6 \pm 0,03$ & $96,7 \pm 5,8$ & $32,1 \pm 3,4$ & $1,7 \pm 0,1^{\mathrm{ab}}$ \\
D-50:9 & $2,2 \pm 0,3$ & $21,5 \pm 1,8^{\mathrm{b}}$ & $4,25 \pm 0,3^{\mathrm{b}}$ & $1,6 \pm 0,004$ & $93,3 \pm 3,3$ & $32,6 \pm 0,9$ & $1,4 \pm 0,2^{\mathrm{a}}$ \\
D-50:12 & $2,3 \pm 0,4$ & $21,4 \pm 2,5^{\mathrm{b}}$ & $4,17 \pm 0,1^{\mathrm{b}}$ & $1,6 \pm 0,04$ & $98,9 \pm 1,9$ & $31,1 \pm 1,8$ & $1,2 \pm 0,2^{\mathrm{a}}$ \\
D-50:15 & $2,4 \pm 0,3$ & $20,4 \pm 2,3^{\mathrm{b}}$ & $4,03 \pm 0,3^{\mathrm{b}}$ & $1,6 \pm 0,10$ & $97,8 \pm 1,9$ & $32,2 \pm 3,2$ & $1,6 \pm 0,2^{\mathrm{a}}$ \\
ANDEVA de dos vías & & & & & & & $0,0,0$ \\
Nivel de proteína & & 0,0001 & 0,0137 & 0,1803 & 0,0852 & 0,4728 & 0,0001 \\
Nivel de lípidos & & 0,0763 & 0,716 & 0,9615 & 0,9147 & 0,1490 & 0,0137 \\
Nivel proteínas x lípidos & & 0,2910 & 0,6690 & 0,5839 & 0,0683 & 0,0540 & 0,2934 \\
\hline
\end{tabular}

P: \% de proteína; L: \% de lípidos; PI: Peso inicial; IP: Incremento en peso; TEC: Tasa especifica de crecimiento; S: Supervivencia; CA: Consumo de alimento; TCA: Tasa de conversión alimenticia

alimentados con las dietas de $40 \%$ de proteína y sus tres niveles de lípidos. Por otro lado, se observó que el nivel de proteína o lípidos en la dieta no afectó significativamente $\left(\mathrm{F}_{(2,18)}=1,88, P=0,18\right)$ el K y se obtuvo un valor promedio de $1,6 \pm 0,04$. La supervivencia obtenida en todos los tratamientos fue mayor al 90\%, excepto en la dieta de 40P:15L que presentó una supervivencia de 86\%, pero no fue significativamente menor $\left(\mathrm{F}_{(2,18)}=2,83, P=\right.$ $0,08)$ al resto de los tratamientos.

Se observó que el porcentaje de proteína y de lípidos en la dieta no afectó significativamente $\left(\mathrm{F}_{(2,18)}=0,78, P=\right.$ 0,47 ) el consumo de alimento; sin embargo, se observaron diferencias significativas en la TCA, los peces alimentados con la dieta 40P:15L presentaron una TCA significativamente mayor $\left(\mathrm{F}_{(2,18)}=17,45, P=0,0001\right)$ en relación a los otros tratamientos.

\section{Discusión}

Los resultados obtenidos en este trabajo indicaron que los juveniles de pargo lunarejo, con un peso inicial de 2,2 g, pueden ser alimentados con dietas que contengan 45 a 50\% de proteína y 9 a 12\% de lípidos para obtener buen rendimiento de crecimiento, supervivencia, factor de condición y tasa de conversión alimenticia, bajo las condiciones experimentales en las cuales se llevó a cabo.
García-Ortega (2009) obtuvo un resultado un tanto distinto, determinando que el requerimiento de proteína y lípidos en la dieta para subadultos de pargo lunarejo silvestre ( $110 \mathrm{~g}$ ) fue de $40 \%$ y $12 \%$, respectivamente. Sin embargo, el requerimiento de proteína disminuye conforme aumenta el tamaño corporal (Tacon 1989), por lo tanto, no es extraño que el requerimiento de proteína de subadultos de pargo lunarejo sea más bajo que lo obtenido en el presente estudio. En estudios realizados con otras especies de pargos se han obtenido resultados similares; por ejemplo, Catacutan et al. (2001) y Abbas et al. (2005) determinaron un requerimiento de $40 \%$ de proteína para el pargo rojo de mangle Lutjanus argentimaculatus y $12 \%$ de lípidos en la dieta, en organismos con peso inicial de 24,8 $\pm 0,4$ g. Para juveniles de pargo criollo $L$. analis de 12,2 g, Watanabe et al. (2001) reportaron un requerimiento de 6 a 9\% de lípidos totales en la dieta. Para subadultos (115 g) de pargo rojo L. campechanus. Miller et al. (2005) reportaron un requerimiento de proteína de $35 \%$ y $10 \%$ de lípidos en la dieta.

Se ha demostrado que los peces marinos carnívoros necesitan en sus dietas altos niveles proteicos (entre 40 a $60 \%$ ) y lipídicos (6 a 15\%) para su óptimo crecimiento y supervivencia, e.g., eglefinos Melanogrammus aeglefinus (Kim \& Lall 2001); botete diana, Sphoeroides annulatus (Abdo de la Parra et al. 2006, García-Ortega 2009); mero 
Centropristis striata (Alam et al. 2009); Epinephelus malabaricus (Tuan \& Williams 2007); robalo Centropomus undecimalis (Gracia-López et al. 2003); brótola Urophycis brasiliensis (Bolasina \& Fenucci 2007); lenguado japonés Paralichthys olivaceus (Lee \& Kim 2005) lenguado del sur P. lethostigma (Gao et al. 2005); besugo Pagellus bogaraveo (Silva et al. 2006); cabrilla arenera Paralabrax maculatofasciatus (Álvarez-González et al. 2001); atún aleta azul Thunnus orientalis (Biswas et al. 2009) y varias especies de Lutjanus (Watanabe et al. 2001, Miller et al. 2005, García-Ortega 2009, presente estudio).

Aún cuando los porcentajes proteicos y lipídicos en la dieta, evaluados en el pargo lunarejo, son similares a los reportados para otras especies congenéricas, las TEC obtenidas en el presente estudio fueron mayores a las determinadas para esas especies; por ejemplo, para Lutjanus analis se reportaron TEC aproximados de 0,84 a 1,25 en juveniles con peso inicial de 12 g y alimentándolos con dietas de 6 a 15\% de lípidos (Watanabe et al. 2001); para juveniles de L. argentimaculatus de 8 y 27,1 g, Abbas et al. (2005) y Abbas \& Siddiqui (2009) reportaron TEC cercanas a 2,5 , alimentando a los peces con dietas con $40 \%$ de proteína. Para la misma especie, Catacutan et al. (2001) reportaron TEC aproximados de 1,8 alimentando a juveniles de $21,0 \mathrm{~g}$ con dietas que contenían $44 \%$ de proteína y $10 \%$ de lípidos. Las altas TEC encontradas en este estudio se deben principalmente a la talla inicial de los organismos experimentales, ya que el crecimiento de los peces, es rápido al principio y luego decrece suavemente; este comportamiento puede representarse mediante una curva sigmoidea (Morales-Nin 1991).

Otra causa de los altos valores de TEC obtenidos para el pargo lunarejo, puede estar relacionada con el origen de la fuente de proteína de las dietas experimentales; en el presente estudio, las fuentes proteicas fueron exclusivamente de origen animal (harina de pescado y caseína), mientras que en otros trabajos las dietas fueron elaboradas también con fuentes proteicas de origen vegetal (pasta de soya, harina de trigo, harina de arroz y tapioca) y tal vez no contenían un balance adecuado de aminoácidos. Es posible que además, estas dietas no sean muy digestibles para los pargos, debido a que los peces carnívoros presentan una limitada habilidad para digerir carbohidratos (Webster \& Lim 2002).

Los lípidos, por su parte, son la principal fuente de energía y de ácidos grasos esenciales para el crecimiento y desarrollo de los peces marinos, pero no son capaces de alargar la cadena y desaturar el ácido linolénico (Tacon 1989, Webster \& Lim 2002); por lo que, es necesario suplementar las dietas con ácido eicosapentaenoico (EPA) y ácido docosahexanoico (DHA) en concentraciones de 0,8 a 2\% (NRC 1993). En el presente estudio, los lípidos de las dietas experimentales provenían de la harina de pescado y del aceite de hígado de bacalao, los cuales contienen más de 20\% de EPA y DHA; por lo cual, probablemente la inclusión de al menos 9\% de lípidos totales derivados de ambas fuentes, en las dietas evaluadas, suplieron los requerimientos de EPA y DHA de los pargos. Sin embargo, se necesitan futuros experimentos para determinar el requerimiento de estos ácidos grasos de los juveniles de pargo lunarejo.

El factor de condición (K), que expresa el estado fisiológico en términos numéricos, no presentó diferencias significativas entre los tratamientos evaluados en el presente estudio, por lo que consideramos que los niveles de proteína y lípidos probados son suficientes para no afectar el K de los juveniles de pargo lunarejo. Resultados similares reportaron Catacutan et al. (2001) en juveniles de 24,8 g y Abbas et al. (2005) en organismos de $8 \mathrm{~g}$ del pargo rojo de manglar (Lutjanus argentimaculatus).

En este trabajo se obtuvieron TCA de 1,2 a 2,3, valores mejor considerados a los reportados por Villa-López (2005) para subadultos de L. guttatus, quien obtuvo TCA de 2,4 a 4,4 y a los obtenidos en estudios de juveniles de otras especies de pargos, alimentados con diferentes niveles de proteína y lípidos con valores de TCA de 2,17 a 3,98 (Catacutan et al. 2001, Watanabe et al. 2001). Estas diferencias pueden ser atribuidas, al origen de la fuente de proteínas de las dietas experimentales de cada estudio y a la talla inicial de los peces.

En base a los resultados obtenidos en el presente estudio, se recomienda incluir por lo menos $45 \%$ de proteína y $9 \%$ de lípidos en las dietas de juveniles de pargo lunarejo. Estos resultados serán de gran utilidad para contribuir en la formulación de dietas prácticas nutricionalmente balanceadas para el pargo lunarejo y coadyuvar en el desarrollo tecnológico del cultivo de esta especie tan apreciada en el mercado mexicano.

\section{Agradecimientos}

Los autores agradecen a la MPA. N. García-Aguilar por proporcionar los huevos de pargo para obtener los juveniles, a la M.C. G. Velasco-Blanco por proporcionar el alimento vivo para el cultivo larvario; a la biol. V. P. Domínguez-Jiménez por los análisis de calidad de agua, a V. Williams por su ayuda en el inglés y por la revisión del documento y la asistencia técnica de A. Ibarra, M. Cruz y 
J. Huerta. Este trabajo fue financiado por el Fondo Mixto Sinaloa (FOMIX) SIN-2006 C01- 37105 dirigido por M.I. Abdo de la Parra.

\section{LITERATURA CITADA}

Abbas G \& PJA Siddiqui. 2009. Effects of different feeding level on the growth, feed efficiency and body composition of juvenile mangrove red snapper, Lutjanus argentimaculatus (Forsskal 1775). Aquaculture Research 40: 781-789.

Abbas G, K Jamil, R Akhtar \& K Hong. 2005. Effects of dietary protein level on growth and utilization of protein and energy by juvenile mangrove red snapper (Lutjanus argentimaculatus). Journal of Ocean University of China 4(1): 49-55.

Abdo de la Parra MI, JL Camacho, B González-Rodríguez, I Martínez-Rodríguez, C Hernández \& A GarcíaOrtega. 2006. A preliminary study on the effect of dietary protein level on growth and survival of juvenile bullseye puffer Sphoeroides annulatus. World Aquaculture 3(1): 3437.

Abdo de la Parra MI, LE Rodríguez-Ibarra, F CampilloMartínez, G Velasco-Blanco, N García-Aguilar, LS Álvarez-Lajonchère \& D Voltolina. 2010. Efecto de la densidad de siembra sobre el crecimiento y supervivencia larval del pargo lunarejo Lutjanus guttatus (Steindachner, 1869). Revista de Biología Marina y Oceanografía 45(1): 141-146.

Alam MS, A Wade, O Watanabe, PM Carroll \& T Rezek. 2009. Effects of dietary protein and lipid levels on growth performance and body composition of black sea bass Centropristis striata (Linnaeus 1758) during grow-out in a pilot-scale marine recirculating system. Aquaculture Research 40: 442-449.

Álvarez-González CA, R Civera-Cerecedo, JL OrtizGalindo, S Dumas, M Moreno-Legorreta \& T GrayebDel Álamo. 2001. Effect of dietary protein level on growth and body composition of juvenile spotted sand bass, Paralabrax maculatofasciatus, fed practical diets. Aquaculture 194: 151-159.

AOAC. 2000. Official methods of analysis of association of official analytical chemists, $684 \mathrm{pp}$. Association of Official Analytical Chemists, Arlington.

Biswas BK, SC Ji, AK Biswas, M Seoka, YS Kim, KI Kawasaki \& K Takii. 2009. Dietary protein and lipid requirements for the Pacific bluefin tuna Thunnus orientalis juvenile. Aquaculture 288: 114-119.

Bolasina SN \& JL Fenucci. 2007. Effects of dietary lipid level on growth, survival and body composition of Brazilian codling (Urophycis brasiliensis Kaup, 1858). Revista de Biología Marina y Oceanografía 42(1): 23-27.
Boza-Abarca J, E Calvo-Vargas, N Solís-Ortiz \& J Komen. 2008. Desove inducido y crecimiento larval del pargo manchado, Lutjanus guttatus, en la Estación de Biología Marina de Puntarenas, Costa Rica. Ciencias Marinas 34(2): 239-252

Catacutan MR, GE Pagador \& S Teshima. 2001. Effects of dietary protein and lipid levels and protein to energy ratios on growth, survival and body composition of the mangrove red snapper, Lutjanus argentimaculatus (Forsskal, 1755) Aquaculture Research 32: 811-818.

Davis DA, KL Bootes \& CR Arnold. 2000. Snapper (Family Lutjanidae) culture. In: Stickney RR (ed). Encyclopedia of aquaculture, pp. 884-889. John Wiley \& Sons, New York.

Ebling ME. 1968. The Dumas method for nitrogen in feeds. Journal Association of Official Analytical Chemists 51: 766770 .

Gao Y, J Lv, Q Lin \& L Li. 2005. Effect of protein levels on growth, feed utilization, nitrogen and energy budget in juvenile southern flounder, Paralichthys lethostigma. Aquaculture Nutrition 11: 427-433.

García-Ortega A. 2009. Nutrition and feeding research in the spotted rose snapper (Lutjanus guttatus) and bullseye puffer (Sphoeroides annulatus), new species for marine aquaculture. Fish Physiology and Biochemistry 35(1): 69-80.

Gracia-López V, T García-Galano, G Gaxiola-Cortés \& J Pacheco-Campos. 2003. Efecto del nivel de proteína en la dieta y alimentos comerciales sobre el crecimiento y la alimentación en juveniles del robalo blanco, Centropomus undecimalis (Bloch, 1792) Ciencias Marinas 29(4B): 585594.

Ibarra-Castro L \& NJ Duncan. 2007. GnRHa-induced spawning of wild-caught spotted rose snapper Lutjanus guttatus. Aquaculture 272: 737-746.

Kim JD \& SP Lall. 2001. Effect of dietary protein level on growth and utilization of protein and energy by juvenile haddock (Melanogrammus aeglefinus). Aquaculture 195: 311-319.

Lee SM \& KD Kim. 2005. Effect of various levels of lipid exchanged with dextrin at different protein level in diet on growth and body composition of juvenile flounder Paralichthys olivaceus. Aquaculure Nutrition 11: 435-442.

Miller CL, DA Davis \& RP Phelps. 2005. Effects of dietary protein and lipid on growth and body composition of juvenile red snapper Lutjanus campechanus. Aquaculture Research 36: 52-60.

Morales-Nin B. 1991. Determinación del crecimiento de peces óseos en base a la microestructura de los otolitos. FAO Documento Técnico de Pesca 322: 1-58.

National Research Council-NRC. 1993. Nutrient requirements of fish, 115 pp. National Academies Press, Washington. 
Rojas-Herrera AA. 2001. Aspectos de dinámica de poblaciones del huachinango Lutjanus peru (Nicholas y Murphy, 1922) y del flamenco Lutjanus guttatus (Steindachner, 1869) (Pisces: Lutjanidae) del litoral de Guerrero, México. Tesis de Doctorado, Universidad de Colima, Colima, México, 90 pp.

Schulz C, M Huber, J Ogunji \& B Rennert. 2008. Effects of varying dietary protein to lipid ratios on growth performance and body composition of juvenile pike perch (Sander lucioperca). Aquaculture Nutrition 14: 166-173.

Silva P, CAP Andrade, VMFA Timoteo, E Rocha \& LMP Valente. 2006. Dietary protein, growth, nutrient utilization and body composition of juvenile blackspot seabream, Pagellus bogaraveo (Brunnich). Aquaculture Research 37: 1007-1014.

Tacon JA. 1989. Nutrición y alimentación de peces y camarones cultivados. Manual de capacitación. GCP/RLA/ 102/ITA Proyecto Aquila II. FAO Documento de Campo 4: $1-572$.
Tuan LA \& KC Williams. 2007. Optimum dietary protein and lipid specifications for juvenile malabar grouper (Epinephelus malabaricus). Aquaculture 267: 129-138.

Villa-López AY. 2005. Efecto de diferentes niveles de proteína y lípidos en el crecimiento y sobrevivencia del pargo lunarejo Lutjanus guttatus (Steindachner, 1896) (Pisces: Lutjanidae). Tesis de Biología, Facultad de Ciencias del Mar, Universidad Autónoma de Sinaloa, Mazatlán, México, $61 \mathrm{pp}$.

Watanabe WO, SC Ellis \& J Chaves. 2001. Effects of dietary lipid and energy to protein ratio on growth and feed utilization of juvenile mutton snapper Lutjanus analis fed isonitrogenous diets at two temperatures. Journal of the World Aquaculture Society 32: 30-40.

Webster CD \& C Lim. 2002. Nutrients requirements and feeding of finfish for aquaculture, 177 pp. CABI Publishing, Cambridge.

Zar JH. 1996. Biostatistical analysis, 662 pp. Prentice-Hall, Englewood Cliffs.

Recibido el 7 de mayo de 2010 y aceptado el 24 de agosto de 2010 